\title{
Consumer Health Informatics: Past, Present, and Future of a Rapidly Evolving Domain
}

\author{
G. Demiris \\ University of Washington, Seattle, WA, USA
}

\begin{abstract}
Summary
Objectives: Consumer Health Informatics (CHI) is a rapidly growing domain within the field of biomedical and health informatics. The objective of this paper is to reflect on the past twenty five years and showcase informatics concepts and applications that led to new models of care and patient empowerment, and to predict future trends and challenges for the next 25 years.

Methods: We discuss concepts and systems based on a review and analysis of published literature in the consumer health informatics domain in the last 25 years.

Results: The field was introduced with the vision that one day patients will be in charge of their own health care using informatics tools and systems. Scientific literature in the field originally focused on ways to assess the quality and validity of available printed health information, only to grow significantly to cover diverse areas such as online communities, social media, and shared decision-making. Concepts such as home telehealth $\mathrm{mHealth}$, and the quantified-self movement, tools to address transparency of health care organizations, and personal health records and portals provided significant milestones in the field. Conclusion: Consumers are able to actively participate in the decision-making process and to engage in health care processes and decisions. However, challenges such as health literacy and the digital divide have hindered us from maximizing the potential of CHI tools with a significant portion of underserved populations unable to access and utilize them. At the same time, at a global scale consumer tools can increase access to care for underserved populations in developing countries. The field continues to grow and emerging movements such as precision medicine and the sharing economy will introduce new opportunities and challenges.
\end{abstract}

\section{Keywords}

Consumer health, home telehealth, personal health record, patient empowerment

Yearb Med Inform 2016; Suppl1:S42-7

http://dx.doi.org/10.15265//YS-2016-s005

Published online May 20, 2016

\section{Introduction}

In 1993, Tom Ferguson and colleagues organized a conference in Wisconsin, USA, entitled "Consumer Health Informatics: Bringing the Patient Into the Loop". Later the same year, Warner Slack and Tom Ferguson presented a half day tutorial with the same title at the American Medical Informatics Association Annual Meeting in Washington DC. Both the Wisconsin conference and the AMIA manual used for the tutorial included the following prediction: "A new generation of medical computing systems will serve the patient, not just the doctor.... [Experts predict] that these new systems will become an important part of our current effort to reinvent health care, turning patients into providers and providing customized health information at the touch of a button" [1]. In this initiative, Ferguson defined consumer health informatics as "the study, development, and implementation of computer and telecommunications applications and interfaces designed to be used by health consumers" [2]. These were the early days of a new and fast growing subdomain of biomedical and health informatics that emphasized the potential of informatics tools to empower patients, place them at the center of health care processes, equip them with means to engage in effective decision-making, and explore choices. This framework also emphasized the utility of informatics not only for those diagnosed with a condition finding themselves as patients in a health care setting, but rather all health consumers, people who wanted to maintain health and engage in disease prevention and self-management.

When scanning Medline for "consumer health" one finds a total number of 4 articles published in 1992 (none of them using additionally the keyword "informatics") and a total of 86 articles published from 1965 to 1992 . One of the four papers of
1992 is a retrospective on the US President's Committee on Health Education that led to the National Consumer Health Information and Health Promotion Act of 1976 which played a significant part in the development of subsequent national policy initiatives for health promotion and disease prevention [3]. In the early nineties, scientific literature in the consumer health informatics domain focused primarily on the quality of information that consumers may be able to access (mostly in libraries, mass media, and the early versions of web-based health information) in preparation for a clinical encounter or to better understand a disease or treatment plan. There was a pervasive recognition that consumer involvement in individual health care was a growing trend at this time. However, as the review by Entwistle and colleagues pointed out, while "the promotion of consumer involvement in decisions about individual health care is now high on many health policy agendas", the question of "what constitutes quality in information packages (that support consumer involvement) is far from settled" [4].

The same tentative Medline search for "consumer health" revealed a total of 208 articles published almost 25 years later between January and October 2015 (with a subset of 83 using the keyword "informatics") and a total of 3,183 papers since 1965 (an increase of almost 37 times the body of literature in 1992). The focus obviously is much broader than solely quality of consumer information, including topics such as reviewing quality metrics of nursing homes [5], social media [6], genetic testing and personal genomics [7], and the challenge of the digital divide [8]. One of the studies published in 2015 examined various available definitions of consumer health informatics (CHI) and identified 23 studies that provided a $\mathrm{CHI}$ definition [9]. The authors appraised definitions using five criteria (use of published citations, multi-disciplinary 
approach, journal impact, definition comprehensibility, text readability) and concluded that the existing definitions were variable in terms of the quality assessment criteria and that there was a need for continued discussion amongst consumer health informaticians to develop a clear consensus definition of $\mathrm{CHI}$ [9]. Indeed since the definition of Ferguson in 1993, several individuals and groups have provided additional definitions in an effort to capture an ever growing and evolving interdisciplinary field. Gibbons et al defined $\mathrm{CHI}$ in 2009 as "any electronic tool, technology, or electronic application that is designed to interact directly with consumers, with or without the presence of a health care professional that provides or uses individualized (personal) information and provides the consumer with individualized assistance, to help the patient better manage their health or health care" [10].

$\mathrm{CHI}$ experienced rapid growth in the past 25 years. One of the earliest integrated consumer health informatics applications was the Comprehensive Health Enhancement Support System (CHESS) developed by Gustafson and colleagues in 1989 at the University of Wisconsin as a computer-based system of integrated services structured to help individuals manage health crises or medical concerns [11]. In several studies focusing on the efficacy of the CHESS software for women with breast cancer, Gustafson and colleagues demonstrated that it improved participants' social support, participation in health, information competence, and confidence in their health care provider significantly when compared to women who only used the Internet $[11,12]$. Since the initial inception of CHESS, the system has evolved significantly and has been expanded to utilize multiple portable technologies and target multiple health care conditions and populations.

In recognition of the growth of scientific studies in this domain, in 2008, the MeSH term "Consumer Health Information" was introduced, defined as "information intended for potential users of medical and healthcare services". The definition stated that this term had an emphasis on self-care and preventive approaches. In 2008, the AMIA Knowledge in Motion Working Group released a white paper on patient-centered applications, exploring the use of IT to promote disease management and wellness [13]. This report acknowledged that new communication technologies can support a transition from institution-centric to patient-centric applications and it defined key principles and challenges for designers, policy makers, and evaluators of patient-centered technologies for disease management and prevention. The paper reviewed emerging trends and highlighted challenges related to design, evaluation, reimbursement, and usability of patient-centered tools.

The growth of $\mathrm{CHI}$ is also reflected in the emergence of curricular informatics activities as well as patient education initiatives that all recognize the importance of consumer-centered care and consumer empowerment. The notion of the patient being an active participant in the health care process is no longer an unattainable goal but rather a reality in many cases. While we are still facing great challenges in establishing accessible consumer-centered health care services [14], many achievements have demonstrated the role $\mathrm{CHI}$ can play in the design of effective, patient-centered systems.

This paper provides an overview of major milestones and facilitators of the growth of $\mathrm{CHI}$ as well as some of potential barriers and challenges. As we reflect on the past 25 years of the field we see how political, social, legal, and technical developments affect the growth of the field and the research directions that are being pursued. A prediction for the next 25 years of $\mathrm{CHI}$ concludes this review.

\section{Milestones of the Past 25 Years of $\mathrm{CHI}$}

\section{Home Telehealth}

The use of telehealth technologies in home care (home telehealth) has seen a rapid growth since the late 90 s. Patients and their families can use technology to monitor vital signs and symptoms of chronic diseases, transmit the data to a clinical site, and access tailored educational resources or communicate via video with home care providers. The concept of home telehealth supports patient engagement as it requires that patients and their families play an active role in monitoring symptoms, learn more about the disease trajectory, and document lifestyle choices (such as nutrition or overall activity). The technology is also meant to improve access to health care services allowing for a more efficient triaging of events and the oversight of the disease trajectory. For patients with chronic conditions, the use of home telehealth is meant to reduce hospitalizations and allow for early detection and intervention.

The field experienced rapid growth and the body of scientific literature moved from early feasibility studies with very limited sample size in the 90s to large randomized clinical trials (RCTs) such as the IDEATEL study in New York [15], the Telemonitoring Study for COPD in Scotland, UK [16], the Tele-ERA study in Minnesota in the US [17], to name a few of the many large RCTs examining the role of telehealth in managing chronic diseases for home care patients. In an evidence synthesis of the use of telehealth for chronic disease management, Wootton reviewed a total of 141 randomized controlled trials [18], in which 148 telehealth interventions of various kinds had been tested in 37,695 patients. Most studies reported positive effects with no significant differences between the chronic diseases. There were very few studies of cost-effectiveness included in this body of literature. Findings indicate that the evidence of telehealth value in the management of chronic diseases is not yet solid and in some cases contradictory [18].

Home-based monitoring can be classified as active monitoring (where a patient or family member is asked to initiate the use of software or hardware, and in many cases they need to be trained in the use of these technologies) or passive monitoring (where technologies are embedded in the residential infrastructure enabling the assessment of parameters without someone initiating or operating the system at home). When passive monitoring features are integral components of the home, the setting is often referred to as a "smart home." Smart home projects to support independence of older adults or people with disabilities emerged initially as research demonstration projects but they have since grown in number and type of technologies and settings to also offer commercially available sensor-based solutions for home monitoring [19, 20]. 


\section{Personal Health Records}

Personal health records (PHRs) are consumer-centric tools designed to facilitate tracking, management, and sharing of personal health information. The Markle Foundation defines a PHR as "an electronic application through which individuals can access, manage, and share their health information ... in a private, secure, and confidential environment" [21]. There are two types of PHR systems: tethered and standalone. Tethered PHRs are available only to consumers, who are part of a specific healthcare system or insurance network. Standalone PHRs on the other hand are available for use to any consumer, who chooses to register and create an account [22].

The definition of PHRs includes the feature of interactive data exchange (i.e. consumers enter data but can also send or share these data sets with other entities). Patient portals, affiliated with specific organizations, may in some cases be tethered PHRs if they support this bidirectional information flow, or may in other instances be web environments that display and summarize data but do not facilitate consumers' data entry. Patient portals were introduced and adopted by large health care organizations in the late 1990s worldwide but did not emerge as a widespread concept until several years later [23]. In 2006, Microsoft and Google featured their own version of PHR systems. The proliferation of smart phones, wearable tools, and social media also functioned as an impetus for the population to embrace technology in almost all aspects of life including interactions with the health care system. As is the case with many innovative and emerging technologies, such tools may introduce new abilities and features for consumers but may also widen existing disparities. Jhamb and colleagues found in a recent study of portal use by patients visiting four university-affiliated nephrology offices that older patients, African American patients, and patients with lower household income were more likely not to access the patient portal [24].

In the US, the federal government Meaningful Use Incentive program - introduced in 2009 - detailed the requirements of the use of electronic health record systems by hospitals and eligible health care professionals. One of the priorities of the Meaningful Use program is "engagement of patients and families" which led health care practices to investigate and many to adopt the use of PHR systems or patient portals. Alternative approaches to patient engagement include providing direct patient access to clinician notes. A rapidly growing initiative called OpenNotes is examining the effects of enabling patients to view clinician progress notes. In 2010 a demonstration project, funded in part by the Robert Wood Johnson Foundation, led to 105 volunteer physicians at three institutions and three different states in the US to invite about 20,000 patients to read their notes through secure electronic portals [25]. Findings were very encouraging where even after a year most patients reported benefits including greater control over their health and medication adherence, and physicians reported increased patient engagement without significant workflow changes [26]. Numerous medical institutions including large organizations such as the M.D. Anderson Cancer Center, the Mayo Clinic, and the Veteran Health Administration, have decided to implement OpenNotes. In 2015 the OpenNotes movement grew from 20,000 patients to approximately 5 million patients nationwide [27].

\section{mHealth and the Quantified Self}

mHealth (or mobile health) refers to the use of mobile communication devices to facilitate health communication and access to health information, enable delivery of care services, and support clinical decision making. The proliferation of mobile phones contributed to the growth of mHealth in the late $90 \mathrm{~s}$, the emergence of smart phones and portable tablet computers accelerated this growth. mHealth introduced new opportunities for health services and information in industrialized nations. At the same time, given the penetration of mobile phones in low-resource settings, it highlighted the potential for developing countries to improve the capacity of health systems by taking advantage of a platform that is accessible to a growing segment of the population. Global health interventions began utilizing mHealth tools to promote disease prevention and management, treatment adherence, educational interventions for health behavior change, and communication with hard-to-reach populations. It is estimated that in 2015, approximately 500 million of the 1.4 billion worldwide smartphone users used some type of a mobile health care application [28]. As the number of medical or health care related smartphone apps increases, efforts to address challenges of reliability and validity testing and overall regulation are made at both a national and global level.

The Quantified Self(QS) movement which aims to improve various aspects of life and health through recording and reviewing daily activities and biometrics [29] is a fast growing practice of self monitoring in recent years due to technological advances and breakthroughs in miniaturization of wearable and environmental sensors. The QS movement began as the aspiration for enhanced self-understanding through data collection, accountability, and goal setting [29]. As technology advances, the range of physiological parameters and environmental variables that can be measured keeps growing (including vital signs, steps, overall activity, caloric intake, sleep quality, time spent sitting, air quality, humidity, luminosity etc.). The QS movement is driven by the principle of patient engagement. The underlying hypothesis is that daily consumer engagement in tracking wellness and health lifestyle choices via goal setting and tacking keeps consumers engaged, informed, and motivated in the disease prevention/ health care process. Some of the emerging biometric devices can be costly and usually require continuous access to wireless networks to ensure their effectiveness; this can further widen the digital divide for those who cannot afford new technologies or the infrastructure to support them.

While several of these tools have been designed to target the consumer directly, the plethora of information about daily living and physical function can be of benefit as clinicians monitor their patients longitudinally. For clinicians to become engaged in the processes that QS facilitate, there is a need for an infrastructure that supports massive data retrieval and trending technology. Currently, Electronic Health Record (EHR) systems, the primary systems to access patient data for clinicians within their current workflow, are not designed to receive or store this type of data. Efforts are underway to enable patients to upload health tracking technology data (from 
personal fitness devices such as wearable fitness trackers and smart scales) to patient portals. Clinicians may be hesitant to assume responsibility for a huge amount of new data that would be made available to them without specific guidelines as to how to incorporate new information into decision making around diagnosis and treatment, and without clarity in terms of accountability and liability. On the other hand, effective algorithms and data mining tools can provide aggregate information about trends that indicate proactive interventions. Daily measurements that QS facilitates can provide tailored monitoring of patients with chronic diseases and information about activity, nutrition, and overall physical function can lead to individualized behavior change interventions.

\section{Consumer Genomics}

Direct to consumer (DTC) genetic testing became available in early 2000 and companies entered the market providing products that allowed individual consumers to obtain information about genetic predisposition to diseases and traits [30,31]. The US Food and Drug Administration (FDA) ordered in 2013 the DTC genetic testing company 23andMe to stop offering health-related genetic risk information to new customers [27]. The company complied with this ruling but offered to disclose to new customers only information on genetic ancestry that does not fall under the purview the FDA. In the European Union, the safety of DTC genetic tests entering the EU market is covered by Directive 98/79 on IVD medical devices [31]. While individual EU member states have provided national legislations to control DTC genetic tests, the EU wide directive has had no practical impact on the offering of such tests [31]. Several initiatives are under way to address these new challenges in the EU as well as in other parts of the world. It becomes clear that technological advances and consumer expectations have created a new reality that introduces ambiguity as to what constitutes a medical test or device and what the consequences of direct to consumer targeting may be; the legislative system is trying to catch up.

In the same context, nutrigenetic testing is introduced as a lifestyle product and an alternative to medical genetic tests whereby companies offer advice on lifestyle changes [32]. As Saukko et al [32] argue, the label of 'lifestyle products' recognizes the severity of a genetic test but negotiates for a "hybrid or compromise category" standing "between medicine and consumer culture" [32].

\section{Transparency}

Worldwide efforts are being made to assure that consumers have sufficient information about the quality of care they have received and the performance of health care sites and professionals when they make a decision about treatment, provider, or health care plan choice.

The European Parliament and Council adopted a Directive in 2010 on patients' rights in cross-border health care [33]. As the first harmonization Directive concerning health care, this may be regarded as a legislative milestone for the European Union [33]. It addresses patients' rights that concern transparency and accountability. The Directive includes Article 4(2) that states that "health-care providers shall provide 'relevant information' to help patients make 'an informed choice on treatment options, on the availability, quality and safety of the health care they provide in the Member State of treatment, clear invoices and clear information on prices', as well as in relation to the status of the health-care provider and information pertinent to liability" [33].

Similarly, in the United States the Affordable Care Act of 2010 promotes greater public reporting of health care outcomes. Most public reports provide a comparison of health care providers in terms of quality or cost to help consumers decide where or from whom to seek care. These reports, however, are largely underutilized by consumers and potential explanations may include that the information is difficult to understand or not perceived as relevant to consumers [34, 35].

Consumers are provided with access to information that is meant to facilitate decision-making and provide an overview of choices. While this is a necessary step as part of the patient empowerment framework, it also introduces challenges such as the varying degrees of health literacy and numeracy, consumers' ability to effectively compare statistical and other information and highlights the need for effective, reliable, and valid representation of data sets including meaningful visualizations of data and trends. Many of the existing public reports are designed based on the assumption that patients can accurately interpret quality metrics. It may be the case that consumers' information needs are slightly different and are not sufficiently met by current public reporting efforts (e.g., rather than choosing between providers of a specific procedure, patients may be more interested in identifying the right treatment or procedure given their condition, social and family support, and financial status [35]).

\section{The Next 25 Years of CHI}

While it is hard to predict hardware and software developments in general and new technological discoveries, there are significant new trends on the horizon pertaining to how we understand and deliver health care and what the roles of various stakeholders will be in this new context, and we can predict the significant role $\mathrm{CHI}$ will play in these movements/developments and the important research questions this domain will aspire to answer in the next 25 years.

\section{Precision Medicine}

Precision medicine, namely "prevention and treatment strategies that take individual variability into account [36]" has been identified as a research priority and an emerging reality that will revolutionize health care and disease prevention. Large scale biologic databases, advance computational tools to mine and analyze large data sets, and the use of proteomics, metabolomics, and genomics to better understand individual patients and populations are all unleashing the potential to tailor disease treatment and prevention taking into account individual variability in genes, environment, and lifestyle [36].

Several scientists and policy makers have emphasized the need to train the next generation of scientists to design and implement innovative approaches for detecting and analyzing a wide range of biomedical information including molecular, genomic, cellular, clinical, behavioral, and environ- 
mental parameters [36]. Several initiatives have been implemented to train clinicians in understanding big data and be able to better interpret information that is generated in the context of precision medicine. While these new opportunities require training for new skills and workflow adjustments by stakeholders such as clinicians, academic researchers, and policy makers, it is the patient or more broadly health consumers, who will need effective tools to navigate this new reality and make sense of complex information in order to engage in decision making. In the next several years, we will hopefully witness the design of new CHI tools that will allow consumers to more easily access and interpret all parameters as well as provide their own information needs and preferences, report on lifestyle patterns and priorities, and engage in carefully assigning utilities to various outcomes and options. CHI researchers will be addressing challenges such as how to effectively display and process complex information that takes into account various parameters in order to individualize treatment and prevention, how to solicit consumers' preferences when faced with multiple choices, how to protect privacy and confidentiality while involving multiple stakeholders in the data gathering and decision-making process, and finally how to facilitate access to tailored prevention strategies that are available on an ongoing basis to the health consumer and not only periodically during clinical encounters.

\section{The Sharing Economy}

A recent trend in many countries, facilitated in part by smart phone technology, was the shift from regulated and controlled environments of transactions to a decentralized one where resources are shared among consumers based on trust facilitated through feedback, community networking, and optimization of resources [37]. Such examples include Uber Technologies Inc, an international transportation network company that operates a mobile app which allows consumers to request a trip and have this request route to drivers, who are part of the network and use their own cars. Another example is Airbnb which is a marketplace platform model that connects hosts and travelers facilitating transactions between them without the company actually owning or maintaining any rooms. Such applications disrupt the existing market and create new sources of supply relying on participants' ongoing feedback for developing quality.

One of the early signs of the impact of the shared economy in health care is the re-emergence of the house call. In many countries home visits accounted for the majority or at least a significant component of medical encounters in the beginning of the $20^{\text {th }}$ century but have dropped to significantly lower levels (by 1980 , home visits accounted for $0.6 \%$ of all medical visits in the US [38]). Now however, there are numerous mobile apps based on the idea of a health care provider visiting a patient when and where needed. Pager, for example, is an app developed by one of the Uber co-founders that helps consumers find a doctor within a guaranteed two hour window whereby the doctor will visit the patient's home, office, or hotel room. Similarly, Mend, located in Texas, US, arranges for urgent care calls in one's home. Similar services are delivered by companies like@mendathome or @HealApp.

Other examples of sharing economy apps utilize crowd sourcing to support diagnosis processes.@Crowdmed is a crowd-sourced diagnostic service. As their website states, this application helps "people to overcome obstacles and silos that exist within the medical establishment - empowering patients and assisting doctors who simply cannot know everything about every medical condition" [39]. The system creates a "Medical Detective community" that gets called upon to identify rare and complex conditions [38]. Figure1 is a company based in Toronto that has created an app that allows medical professionals to upload medical images and seek input on complex cases, becoming "Medicine's Instagram" [40].

The sharing economy principles in health care may lead to a radical decentralization of services. Unlike the existing paradigm where specialized professionals provide highly priced services placed in and supervised by large and complex institutions that facilitate access to expensive equipment and other resources, the sharing economy model brings these professionals to people's homes. The introduction of the Uber car rides was met with resistance and concern in many countries, and questions were raised around regulating this new business and mandating standards for training and safety. Such concerns will be only increased when we think about pushing boundaries in the health care world. The consumer preferences for accessible and timely care, and the need for innovation will drive these changes. At the same time, questions around reimbursement and continuity of care will be raised. How viable is a health care organization that is using supply and demand matching systems? Are health care professionals trained and ready for new models of care? How do we ensure patient safety and privacy and proactively address unintended consequences? CHI researchers will be called to inform the design, implementation, and evaluation of appropriate apps and other tools that will maximize the benefits and reduce the risk of a sharing economy model. Ethical, practical, and legal concerns will have to be addressed and the concepts of patient empowerment and autonomy will have to be examined. The work of Kaplan [41], Brennan [42], Derse [43], Miller [44], Wetter [45], and others on the ethical implications of $\mathrm{CHI}$ can and should provide the foundation for such considerations.

The sharing economy has the potential to improve access to health care and the overall quality of the health care experience for consumers and their families. However, a careful balance between innovation and consumer demands on the one hand, and regulatory and quality safeguards on the other, should guide us as we explore this new era.

\section{Conclusion}

The field of $\mathrm{CHI}$ has experienced significant growth in the last 25 years. Inspired by Ferguson's original prediction for $\mathrm{CHI}$ and expanding on that vision, we predict that consumer informatics tools of the future will serve as a personalized information source and decision aid to facilitate tailored monitoring of wellness, disease prevention, and treatment for informed and engaged consumers. CHI tools will facilitate continuity of care and can bridge clinical care, health behavior change, information management and decision-making.

As we embrace innovation and redesign systems and processes based on new IT 
capabilities, we need to be cognizant of the fact that these new tools may further isolate those, who have limited health literacy and/ or limited access to technological resources, namely underserved populations, widening the existing gap. CHI tools, if supported by legislative and public health initiatives, can actually serve as a catalyst to eliminate some of these inequalities and provide support for marginalized populations.

\section{References}

1. Ferguson T. Doctom's FAQ. [cited 2015 November 1]. Available from: http://www.fergusonreport. com/articles/tfr07-03.htm

2. Ferguson T. What is consumer health informatics? In: Ferguson T, ed. The Ferguson Report. Austin; 2001.

3. Guinta MA, Allegrante JP. The President's Committee on Health Education: a 20-year retrospective on its politics and policy impact. Am J Public Health 1992;82(7):1033-41.

4. Entwistle VA, Sheldon TA, Sowden AJ, Watt IS. Supporting consumer involvement in decision making: what constitutes quality in consumer health information? Int J Qual Health Care 1996;8(5):425-37.

5. Liu D, Lu CJ. Visibility and findability of the nursing home compare website. Soc Work Public Health 2015;30(2):144-56.

6. Staccini P, Fernandez-Luque L. Health Social Media and Patient-Centered Care: Buzz or Evidence? Findings from the Section "Education and Consumer Health Informatics" of the 2015 Edition of the IMIA Yearbook. Yearb Med Inform 2015;10(1):160-3.

7. Servick K. Can 23 andMe have it all? Science 2015;349(6255):1472-4, 1476-7.

8. Hall AK, Bernhardt JM, Dodd V, Vollrath MW. The digital health divide: evaluating online health information access and use among older adults. Health Educ Behav 2015;42(2):202-9.

9. Flaherty D, Hoffman-Goetz L, Arocha JF. What is consumer health informatics? A systematic review of published definitions. Inform Health Soc Care 2015;40(2):91-112.

10. Gibbons MC, Wilson RF, Samal L, Lehmann CU, Dickersin K, Lehmann HP, et al. Consumer health informatics: results of a systematic evidence review and evidence based recommendations. Transl Behav Med 2009;1:72-82.

11. Gustafson DH, Hawkins R, Pingree S, McTavish F, Arora NK, Mendenhall J, et al. Effect of computer support on younger women with breast cancer. J Gen Intern Med 2001;16:435-45.

12. Gustafson DH, Hawkins R, Mctavish F, Pingree S, Chen WC, Volrathongchai K, et al. Internet-based interactive support for cancer patients: are integrated systems better? J Commun 2008;58:238-57.

13. Demiris G, Afrin LB, Speedie S, Courtney KL, Sondhi M, Vimarlund V, et al. Patient-centered applications: use of information technology to promote disease management and wellness. A white paper by the AMIA knowledge in motion working group. J Am Med Inform Assoc 2008;15(1):8-13.

14. Gibbons MC, Wilson RF, Samal L, Lehmann CU, Dickersin K, Lehmann HP, et al. Consumer health informatics: results of a systematic evidence review and evidence based recommendations. Transl Behav Med 2011;1(1):72-82.

15. Shea S, Weinstock RS, Teresi JA, Palmas W, Starren J, Cimino JJ, e al; IDEATel Consortium. A randomized trial comparing telemedicine case management with usual care in older, ethnically diverse, medically underserved patients with diabetes mellitus: 5 year results of the IDEATel study. J Am Med Inform Assoc 2009;16(4):446-56.

16. Pinnock H, Hanley J, McCloughan L, Todd A, Krishan A, Lewis S, et al. Effectiveness of telemonitoring integrated into existing clinical services on hospital admission for exacerbation of chronic obstructive pulmonary disease: researcher blind, multicentre, randomised controlled trial. BMJ 2013;347:f6070.

17. Takahashi PY, Hanson GJ, Pecina JL, Stroebel RJ, Chaudhry R, Shah ND, et al. A randomized controlled trial of telemonitoring in older adults with multiple chronic conditions: the Tele-ERA study. BMC Health Services Research 2010;10:255.

18 . Wootton R. Twenty years of telemedicine in chronic disease management-an evidence synthesis. J Telemed Telecare 2012;18(4):211-20.

19. Reeder B, Meyer E, Lazar A, Chaudhuri S, Thompson HJ, Demiris G. Framing the evidence for health smart homes and home-based consumer health technologies as a public health intervention for independent aging: a systematic review. Int J Med Inform 2013;82(7):565-79.

20. Demiris G, Thompson HJ. Mobilizing Older Adults: Harnessing the Potential of Smart Home Technologies. Contribution of the IMIA Working Group on Smart Homes and Ambient Assisted Living. Yearb Med Inform 2012;7:94-9.

21. Connecting for Health; A Public-Private Collaborative. The Personal Health Working Group: Final Report. Markle Foundation; 2003.

22. Archer N, Fevrier-Thomas U, Lokker C, McKibbon KA, Straus SE. Personal health records: a scoping review. JAm Med Inform Assoc 2011;18(4):515-22.

23. Halamka JD, Mandl KD, Tang PC. Early experiences with personal health records. J Am Med Inform Assoc 2008;15(1):1-7.

24. Jhamb M, Cavanaugh KL, Bian A, Chen G, Ikizler TA, Unruh ML, et al. Disparities in Electronic Health Record Patient Portal Use in Nephrology Clinics. Clin J Am Soc Nephrol [Epub ahead of print]

25. Leveille SG, Walker J, Ralston JD, Ross SE, Elmore JG, Delbanco T. Evaluating the impact of patients' online access to doctors' visit notes: designing and executing the OpenNotes project. BMC Med Inform Decis Mak 2012;12:32.

26. Delbanco T, Walker J, Bell SK, Darer JD, Elmore JG, Farag N, et al. Inviting patients to read their doctors' notes: A quasi-experimental study and a look ahead. Ann Intern Med 2012;157:461-70.

27. Walker J, Meltsner M, Delbanco T. US experience with doctors and patients sharing clinical notes. BMJ 2015;350:g7785.

28. Global Mobile Health Trends and Figures Market Report 2013-2017. http://research2guidance. com/2010/11/10/500m-people-will-be-usinghealthcare-mobile-applications-in-2015-2/

29. Appelboom G, LoPresti M, Reginster JY, Sander Connolly E, Dumont EP. The quantified patient: a patient participatory culture. Curr Med Res Opin 2014;30(12):2585-7.

30. Prainsack B, Reardon J, Hindmarsh R, Gottweis $\mathrm{H}$, Lunshof JE. Misdirected precaution? Nature 2008;456:34-5

31. Kalokairinou M, Howard HC, Borry P. Changes on the horizon for consumer genomics in the EU. Science 2014;346(6207):296-8.

32. Saukko PM, Reed M, Britten N, Hogarth S. Negotiating the boundary between medicine and consumer culture: online marketing of nutrigenetic tests. Soc Sci Med 2010;70(5):744-53.

33. Delnoij D, Sauter W. Patient information under the EU patients' rights Directive. Eur J Public Health 2011;21(3):271-2.

34. Health Policy Brief: public reporting on quality and costs. Health Affairs; March 8, 2012.

35. Huckman RS, Kelley MA. Public Reporting, Consumerism, and Patient Empowerment. The New Engl J Med 2013;369(20):1875-7.

36. Collins FS, Varmus H. A new initiative on precision medicine. N Engl J Med 2015;372(9):793-5.

37. Cohen B, Kietzmann J. Ride On! Mobility Business Models for the Sharing Economy. Organization \& Environment 2014;27(3):279-96.

38. Meyer GS, Gibbon RV. House Calls to the Elderly-A Vanishing Practice Among Physicians. New Engl J Med 1997;337:1815-20.

39. CrowdMed. [cited November 1, 2015] Available from: https://www.crowdmed.com/our-inspiration

40. Wall Street Journal: Photo Sharing Without LolCats: Figure1 raises $1 \mathrm{M}$ to become Medicine's Instagram. [cited November 1, 2015] Available from: http://blogs.wsj.com/venturecapital/2014/08/06/ photo-sharing-without-lolcats-figure-1-raises-4m-to-become-medicines-instagram/

41. Kaplan B. Selling health data: de-identification, privacy, and speech. Camb Q Healthc Ethics 2015;24(3):256-71.

42. Brennan PF, Downs S, Casper G. Project HealthDesign: rethinking the power and potential of personal health records. J Biomed Inform 2010;43(5 Suppl):S3-5.

43. Derse AR, Miller TE. Net effect: professional and ethical challenges of medicine online. Camb Q Healthc Ethics 2008 Fall;17(4):453-64.

44. Miller TE, Horowitz CR. Disclosing doctors' incentives: will consumers understand and value the information? Health Aff (Millwood) 2000;19(4):149-55.

45. Wetter T. Consumer Health Informatics. New Services, Roles, and Responsibilities. London: Springer; 2015.

\section{Correspondence to:}

George Demiris PhD, FACMI

Professor

University of Washington

BNHS Box 357266

Seattle, WA 98195, USA

Tel: + 12062213866

E-mail:gdemiris@uw.edu 